\title{
Filler effects on the thermomechanical response of stretched rubbers
}

\author{
J.R. Samaca Martinez ${ }^{\mathrm{a}, \mathrm{b}, \mathrm{c}}$, J-B. Le Cam ${ }^{\mathrm{d}, *}$, X. Balandraud $^{\mathrm{b}, \mathrm{e}}$, E. Toussaint ${ }^{\mathrm{a}, \mathrm{b}}$, \\ J. Caillard ${ }^{\mathrm{c}}$ \\ ${ }^{a}$ Clermont Université, Université Blaise Pascal, Institut Pascal, BP 10448, 63000 Clermont-Ferrand, France \\ ${ }^{\mathrm{b}}$ CNRS, UMR 6602, Institut Pascal, 63171 Aubière, France \\ ${ }^{\mathrm{c}}$ Michelin, CERL Ladoux, 63040 Clermont-Ferrand, France \\ d Université de Rennes 1, Larmaur ERL CNRS 6274, Campus de Beaulieu, 35042 Rennes, France \\ ${ }^{\text {e } C l e r m o n t ~ U n i v e r s i t e ́, ~ I n s t i t u t ~ F r a n c ̧ a i s ~ d e ~ M e ́ c a n i q u e ~ A v a n c e ́ e, ~ I n s t i t u t ~ P a s c a l, ~ B P ~ 10448, ~} 63000$ Clermont-Ferrand, France
}

This paper deals with the calorimetric analysis of deformation processes in filled styrene-butadiene rubbers. More especially, the study focuses on the effects of the addition of carbon black fillers on the calorimetric response of "demullinized" SBR. Temperature variations are measured by infrared thermography during cyclic uniaxial tensile tests at ambient temperature. Heat sources ${ }^{1}$ produced or absorbed by the material due to defor-mation processes are deduced from temperature fields by using the heat diffusion equa-tion. First, the results show that no mechanical (intrinsic) dissipation is detected for weakly filled SBR, meaning that the heat produced and absorbed over one mechanical cycle is the same whatever the stretch ratio reached. Second, the mechanical dissipation in highly filled SBR is significant. The quantitative analysis carried out highlights the fact that it increases quasi-linearly with the stretch ratio. Finally, a simplified framework is pro-posed to discuss the identification of the heat sources, in particular the mechanical dissipation.

\section{Introduction}

Elastomers are widely used in industrial and research applications, mainly due to their ability to undergo large deformations without any damage, and to their damping properties. Nevertheless, due to their poor thermal conductivity, such materials are subject to significant self-heating during stretching, all the more so if they are reinforced by fillers.

In this study, infrared thermography (IRT) is used to investigate the effects of fillers on the thermomechanical

\footnotetext{
* Corresponding author. Tel.: +33 223235741.

E-mail address: jean-benoit.lecam@univ-rennes1.fr (J.-B. Le Cam).

1 The term heat source is used in this paper to mean the heat power being produced or absorbed.
}

response of styrene-butadiene rubber (SBR). IRT is a full thermal field measurement technique that provides accurate information about temperature variations at the surface of a specimen subjected to influences that can differ in nature (mechanical, thermal, chemical...). The temperature changes are due to the material response (heat source due to thermoelasticity, viscosity, phase changes, straininduced crystallization, damage...) as well as structure effects (heat conduction and heat exchanges with the environment outside the specimen under study). Thus, due to heat conduction and heat exchanges, temperature is not a relevant quantity (see for example [1,2]). For instance, temperature variation does not allow us to determine the characteristic strains at which strong exo- or endothermal phenomena occur, typically chain crystallization and crystallite melting (see for example [3]). 
This is the reason why thermal analysis has widely been extended to quantitative calorimetry (see for instance [4]). For this purpose, the framework of the thermodynamics of irreversible processes (TIP) and the heat diffusion equation are used. This approach enables us to measure the total heat source produced or absorbed by the material. Under certain hypotheses, it is also possible to distinguish the part due to thermomechanical couplings, including thermoelastic couplings, from the part due to mechanical dissipation. This approach is used in the present study in order to determine the variation in mechanical dissipation due to filler effects in a SBR whose mechanical behaviour is stabilized, i.e. "demullinized". The mechanical dissipation here is only due to viscosity, not to the Mullins effect.

The paper is composed of two parts. The first presents the experimental set-up while the second presents the results, analysis and discussion. Concluding remarks close the paper.

\section{Experimental setup}

\subsection{Material and specimen geometry}

The materials considered here were SBR filled with two different amounts of N347 carbon black, 5 and 50 phr (part per hundred of rubber in weight), they are denoted SBR5 and SBR50, respectively. As shown in Table 1, apart from the filler quantity, their formulations were the same. The molar mass and the glass transition temperature of the SBR used were respectively $120,000 \mathrm{~g} / \mathrm{mol}$ and $-48{ }^{\circ} \mathrm{C}$. It should be noted that these material formulations do not lead to stress-induced crystallization, unlike natural rubber, for instance. SBR5 and SBR50 were cured for 35 and $22 \mathrm{~min}$, respectively. The mould temperature was set to $150^{\circ} \mathrm{C}$.

The non-standard specimen geometry is presented in Fig. 1. It was a thin dumbbell-shaped specimen, with width, length and thickness equal to $5 \mathrm{~mm}, 10 \mathrm{~mm}$ and $1.4 \mathrm{~mm}$, respectively. It can be noted that the width was chosen to ensure the homogeneity of the mechanical fields during uniaxial tensile tests, i.e. a uniaxial tension state.

\subsection{Loading conditions}

The mechanical loading corresponded to uniaxial cyclic loading. It was applied under prescribed displacement using an INSTRON 5543 testing machine. The signal shape was triangular in order to ensure a constant strain rate during loading and unloading. The loading and nominal strain rates were equal to $\pm 300 \mathrm{~mm} / \mathrm{min}$ and $\pm 0.5 \mathrm{~s}^{-1}$,

Table 1

Chemical composition in parts per hundred rubber (phr).

\begin{tabular}{lcc}
\hline Ingredient & SBR5 & SBR50 \\
\hline Styrene-Butadiene rubber (SBR) & 100 & 100 \\
Carbon black & 5 & 50 \\
Antioxidant 6PPD & 1.9 & 1.9 \\
Stearic acid & 2 & 2 \\
Zinc oxide ZnO & 2.5 & 2.5 \\
Accelerator CBS & 1.6 & 1.6 \\
Sulfur solution 2H & 1.6 & 1.6 \\
\hline
\end{tabular}

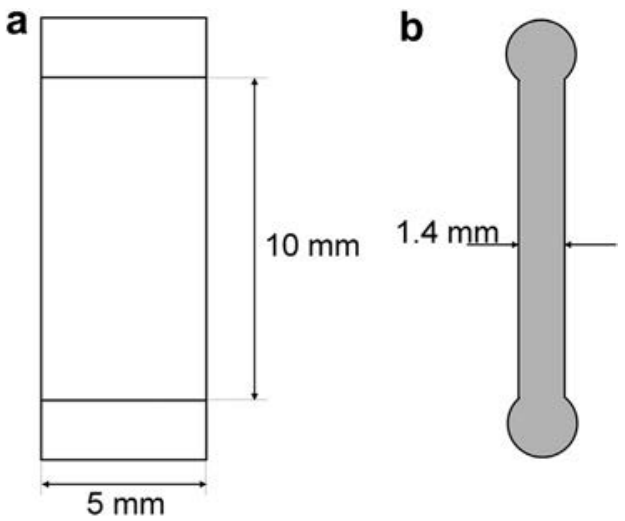

Fig. 1. Specimen geometry: (a) front view; (b) side view.

respectively. The test corresponded to series of three cycles at four increasing maximum stretch ratios, defined as the ratios between the current and the initial length of the specimen. The maximum stretch ratio levels were chosen as follows:

- For the SBR5, the four maximum stretch ratios were $\lambda_{1}=2, \lambda_{2}=3, \lambda_{3}=3.5$ and $\lambda_{4}=4$, as shown in Fig. 2(a).

- For the SBR50, the four maximum stretch ratios were $\lambda_{1}=2, \lambda_{2}=3, \lambda_{3}=4$ and $\lambda_{4}=4.5$, as shown in Fig. 2(b).

$\lambda_{3}$ and $\lambda_{4}$ slightly differed from one formulation to the other, due to the fact that the elongations at failure were different (4.2 for SBR5 and 4.8 for SBR50).

\subsection{Temperature field measurement}

Temperature field measurements were performed using a Cedip Jade III-MWIR infrared camera, which features a local plane array of $320 \times 240$ pixels and detectors with a wavelength range of 3.5-5 $\mu \mathrm{m}$. Integration time was equal to $1500 \mu \mathrm{s}$. The acquisition frequency $\mathrm{f}_{\mathrm{a}}$ was $147 \mathrm{~Hz}$. The thermal resolution, namely the noise-equivalent temperature difference, was equal to $20 \mathrm{mK}$ for a temperature range of $5-40{ }^{\circ} \mathrm{C}$. The calibration of the camera detectors was performed with a black body using a Non-Uniformity Correction (NUC) procedure. During the measurement, the external heat sources were reduced by using a black box surrounding the specimen, featuring a small window for the IR camera to be able to observe the gauge zone of the specimen. The thermal quantity considered in the present study was the mean temperature variation of a small zone at the centre of the specimen. This quantity was obtained by subtracting the initial temperature from the current one, after applying a suitable movement compensation technique [3] to track this small zone during the test.

\subsection{Heat source calculation}

The heat sources produced or absorbed by the material itself were studied within the framework of the thermodynamics of irreversible processes. Considering the first and second principles of thermodynamics and assuming 

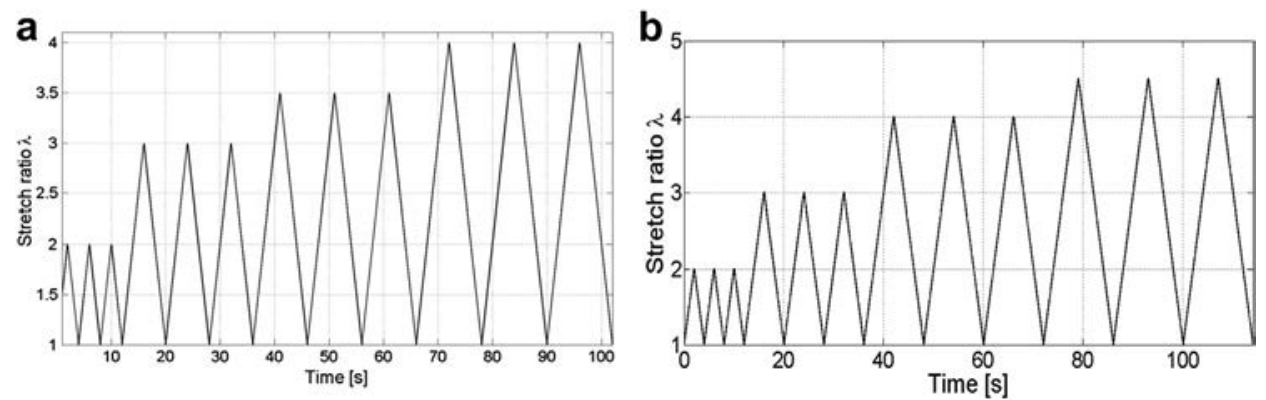

Fig. 2. Mechanical loading conditions: (a) SBR5; (b) SBR50.

Fourier's law to model heat conduction $[5,6]$, the heat diffusion equation is written:

$\rho C_{E, V k} \dot{T}-\operatorname{div}(\operatorname{Kgrad} T)-r=s_{h}$

with

$s_{h}=d_{1}+s_{T M C}$

where:

- $s_{h}$ and $r$ are the heat source produced or absorbed by the material due to stretch and the external heat source (e.g. by radiation), respectively;

- $d_{1}$ and $s_{\mathrm{TMC}}$ are the mechanical dissipation (always positive) and the heat source due to the thermomechanical couplings, respectively. In particular, the latter contains the thermoelastic coupling term mainly composed of so-called entropic coupling. The former is related to any irreversible mechanical phenomenon, such as viscosity or "damage"2;

- $\rho, K$ and $C_{E, V k}$ are the density, the thermal conductivity tensor and the specific heat at constant strain $E$ and internal state variables $V_{k}$, respectively.

This equation applies both in the reference configuration as well as in the current configuration, provided that we give a suitable definition of symbols $r$, div, $K$, grad and $s_{h}$. However, it is only in Lagrangian variables that the total derivative $\dot{T}$ can be calculated as a partial derivative.

The temperature fields were measured at the surface of a flat specimen by an IR camera. As the tests performed were assumed to be homogeneous in terms of strain and stress, and as rubbers have a very low thermal diffusivity, the temperature fields were nearly homogeneous. Therefore, the 3D heat diffusion Equation (1) can be reduced to a "OD" formulation as shown in $[1,4,7]$. In this case, the heat diffusion equation can be rewritten:

$\rho C_{E, V k}\left(\dot{\theta}+\frac{\theta}{\tau}\right)=s_{h}$

where $\theta$ is the temperature variation from the initial temperature (here considered in the unstretched state at the

\footnotetext{
${ }^{2}$ The term damage is used here as a generic term for any irreversible change in the material microstructure, for example crack initiation and growth.
}

beginning of the test). In this equation, $\tau$ is a characteristic time that accounts for the heat exchange with the outside environment. In practice, it can be experimentally determined by identification from a natural return to ambient temperature. For the present experiments, the characteristic time $\tau$ was defined as (40.50-3.25 $\lambda$ ) seconds. It is worth noting that the identification of the function $\tau(\lambda)$ must be performed from several values of $\lambda$, applied in the same configuration as the mechanical tests (same specimen geometry tightened in the jaws of the testing machine used). The following procedure was used. The specimen was first stretched in the machine. It was then heated by contact with a 'hot' piece for a few seconds in order to generate as homogeneous a temperature field as possible. The temperature measurement by IR thermography was performed during the natural return to room temperature. The measurement actually started 1 min later in order to ensure better homogeneity of the temperature in the specimen.

Lastly, the heat source $s_{h}$ is divided by the product $\rho C_{E, V \mathrm{k}}$, leading to a quantity $s$ in ${ }^{\circ} \mathrm{C} / \mathrm{s}$ :

$s=\frac{s_{h}}{\rho C_{E, V k}}=\dot{\theta}+\frac{\theta}{\tau}$

In the following, the ratio $s / \rho C_{E, \mathrm{Vk}}$ will be termed "heat source $s$ " for the sake of simplicity. This equation will be used to calculate the heat source $s$ from the temperature variation $\theta$.

\section{Results}

Fig. 3 presents the nominal stress, defined as the force per unit surface, versus the stretch ratio, for SBR5. This figure shows that mechanical cycles have little effect on the mechanical response, in the sense that neither significant stress softening nor a hysteresis loop are observed. Finally, it should be noted that a residual strain is observed which can reach $10 \%$ for the maximum stretch ratio applied.

Fig. 4 presents the results obtained in terms of the heat source versus the applied stretch ratio, for SBR5. Fig. 4(a), (b), (c) and (d) present the results for $\lambda_{1}=2, \lambda_{2}=3$, $\lambda_{3}=3.5$ and $\lambda_{4}=4$, respectively. The unbroken lines correspond to the heat sources calculated on loading and unloading. The dotted line corresponds to the absolute value of the heat sources on unloading. This curve is 


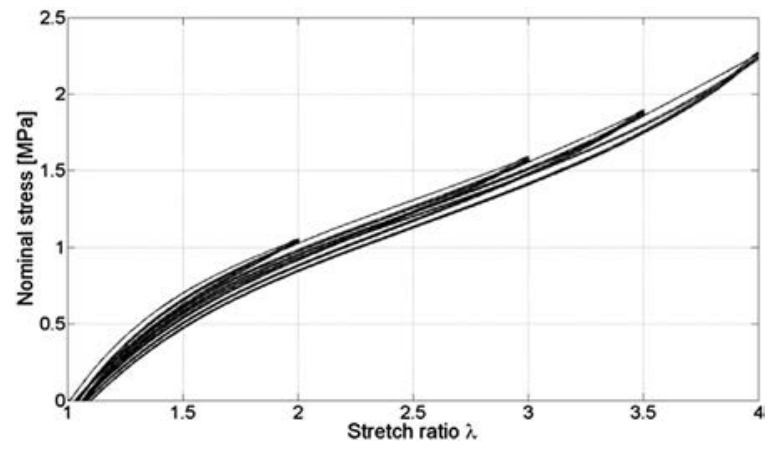

Fig. 3. Mechanical response of SBR5.

plotted in order to compare the heat sources produced and absorbed during loading and unloading. These figures show that the absolute values of the heat sources has the same evolution during loading and unloading, highlighting the fact that no mechanical dissipation is detected. Indeed, mechanical dissipation $d_{1}$ is always a positive quantity, for loading as well as for unloading. If $d_{1}$ had been different from zero during a load-unload cycle, the curves would be asymmetrical. Consequently, the heat sources here are mainly due to thermoelastic coupling. This is corroborated by the fact that no significant mechanical hysteresis is observed in terms of the strain-stress relationship.

In the following, these results are compared to those obtained for SBR50. Results are first discussed qualitatively. They are then analysed quantitatively in order to evaluate accurately the level of mechanical dissipation depending on the maximum stretch ratios applied.

Fig. 5 presents the mechanical response of SBR 50. Each mechanical cycle exhibits a hysteresis loop, and stress softening is observed for each series. This stress softening phenomenon is well known in filled rubbers. It is referred to as "the Mullins effect" in the literature. For further information, the reader can refer to [8-10]. In the present study, the Mullins effect is not investigated. Only the third cycle of each series is considered for our analysis. These cycles are considered as mechanically stabilized in terms of both stress softening and residual strain. It should be noted that no significant increase in residual strain is observed between the second and third cycles, meaning that this residual strain can be seen as a type of "damage" associated with stress softening. It should be noted also that the residual strain reaches 30\% for the maximum stretch ratio applied.

Fig. 6 gives the heat source versus the stretch ratio for each third cycle for SBR50. Fig. 6(a), (b), (c) and (d) present the results for $\lambda_{1}=2, \lambda_{2}=3, \lambda_{3}=4$ and $\lambda_{4}=4.5$, respectively. These figures show that the heat sources produced exhibit a load-unload dissymmetry. This is clearly shown by the comparison between the heat sources produced on loading and the absolute value of the heat source absorbed on unloading. This reveals that mechanical dissipation is produced. Here, the mechanical dissipation is only due to viscosity and not to any "damage". This viscosity is mainly due to the high proportion of filler (50 phr, compared with $5 \mathrm{phr}$ for SBR5).

At this stage of the present study, several remarks can be formulated to analyse the results quantitatively. These remarks concern the mechanical dissipation deduced from
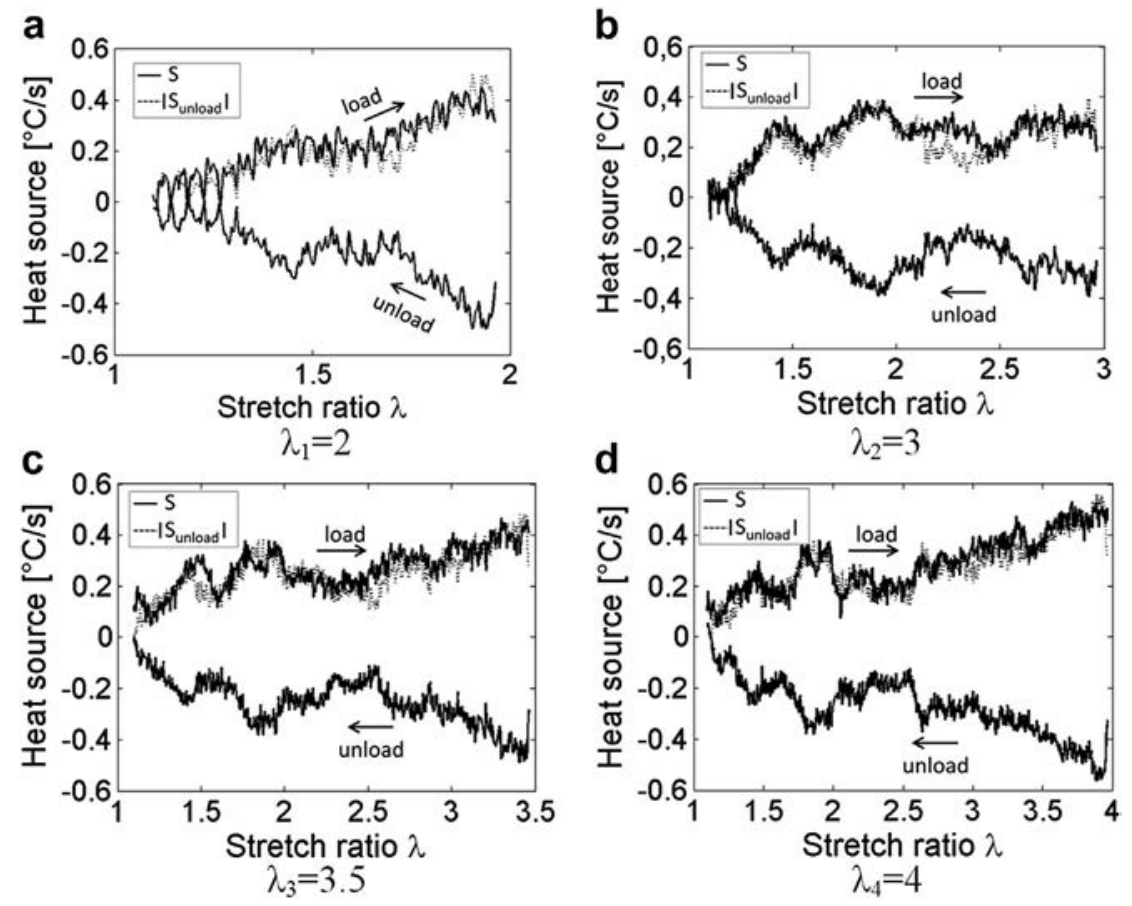

Fig. 4. Heat source versus stretch ratio for the third cycle of each of the four series for SBR 5 . The dotted line corresponds to the absolute value of the heat source during unloading. 


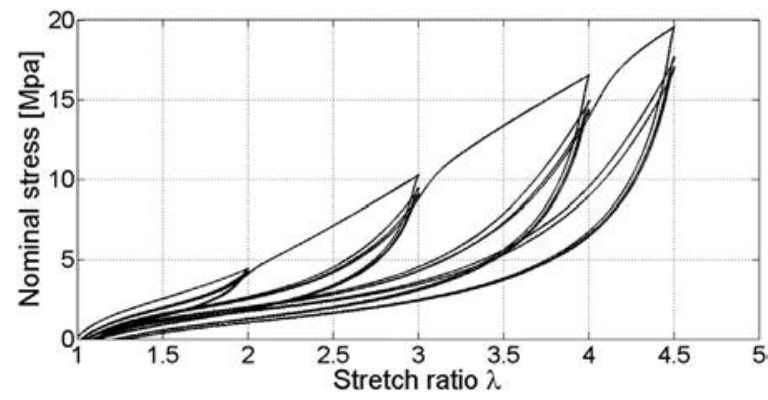

Fig. 5. Mechanical response for SBR50.

the calorimetric response of the material. For this purpose, Fig. 7 gives four different types of diagram that can be obtained according to the nature of the rubber behaviour considered.

The contribution of the physical mechanisms involved in the deformation of rubber to the heat produced at each mechanical cycle leads us to consider various elementary types of calorimetric behaviour, which are illustrated in Fig. 7. Fig. 7(a), (b), (c) and (d) give the calorimetric responses of purely entropic behaviour without mechanical dissipation, with "damage" occurring on loading, with viscosity, and with viscosity and "damage", respectively.

For purely entropic behaviour (Fig. 7(a)), no dissipation occurs during the mechanical cycle. The heat source corresponds to the thermoelastic coupling term only. The variations in the heat source during loading ( $s_{\text {load }}$ ) and unloading $\left(\mathrm{s}_{\text {unload }}\right)$ in absolute value are superimposed.
Consequently, the heat produced on loading is equal to that absorbed during unloading at each stretch ratio considered. This calorimetric response is observed for SBR5, i.e. for weakly filled SBR (see Fig. 4).

Fig. 7(b) gives the response for entropic material with "damage" occurring during loading. This is not the case of Mullins softened SBR50. Obviously, this is more debatable for the first cycle of each series, even if the Mullins effect is mainly observed in filled, and therefore viscous, rubbers.

Fig. 7(c) gives the response for entropic and viscous material. This calorimetric response corresponds to that of Mullins softened SBR50 and provides the modelling of the results shown in Fig. 6. Fig. 7(d) gives the response for entropic and viscous material which is "damaged" during loading. It should be noted that this analysis applies only for the first cycle in filled rubber. The consequences of the Mullins effect on the calorimetric response are studied in a future paper under review for publication.

This interpretation framework, especially Fig. 7(c), leads to a quantitative analysis of the calorimetric response of SBR50 by calculating the difference between the heat produced on loading and that absorbed during unloading. This leads us to calculate first $A_{n}=\int s_{\text {load }} d t$, then $B_{n}=\int s_{\text {unload }} d t$ and finally $C_{n}=\int s_{\text {load }} d t+\int s_{\text {unload }} d t$. All of these quantities are expressed in ${ }^{\circ} \mathrm{C}$. The heat corresponding to the mechanical dissipation over one mechanical cycle $\mathrm{H}_{\mathrm{d} 1}$ (in $10^{6} \mathrm{~J} / \mathrm{m}^{3}$ ) is calculated by multiplying $C_{\mathrm{n}}$ by the product $\rho C_{E, \mathrm{Vk}}$. Table 2 gives the results obtained; they are summarized in the diagram in Fig. 8. As shown in this figure, heat sources absorbed and produced increase quasilinearly with the maximum stretch ratio during stabilized
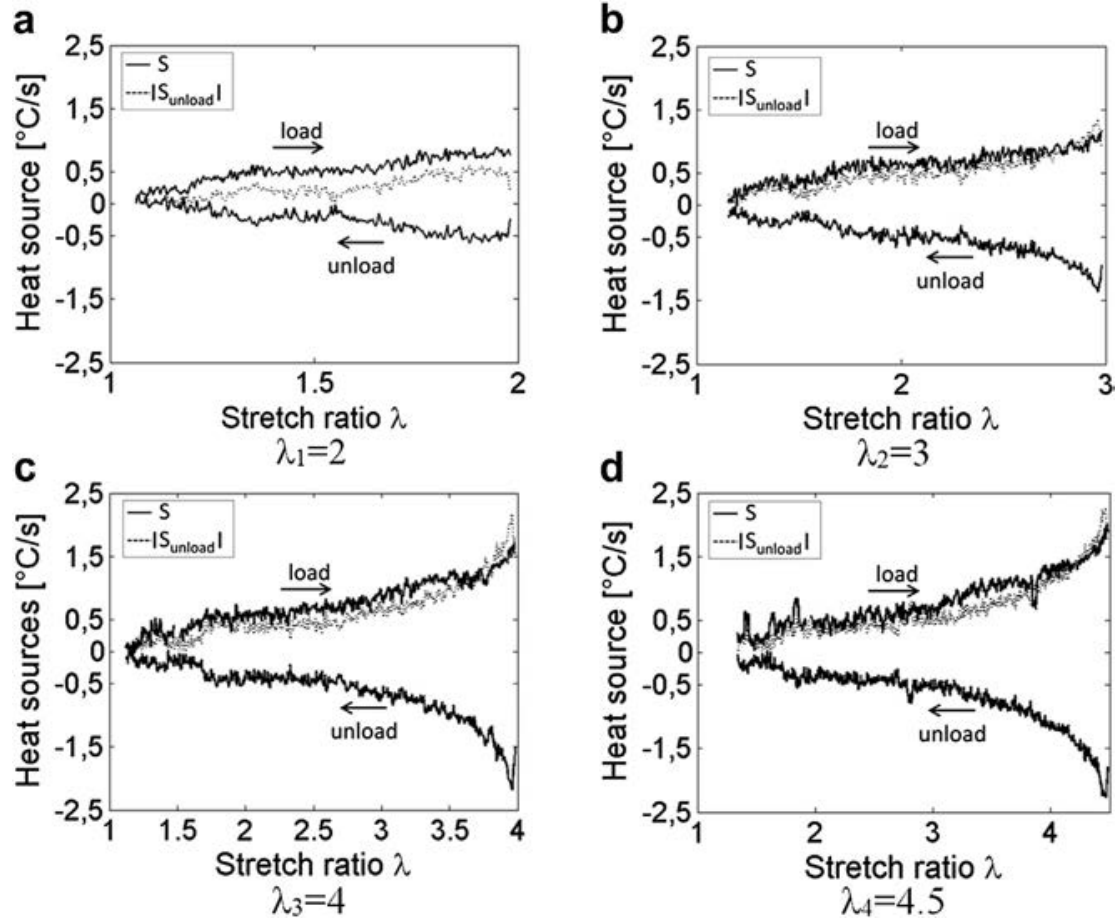

Fig. 6. Heat source versus stretch ratio for the third cycle of each of the four series for SBR 50 . The dotted line corresponds to the absolute value of the heat source during unloading. 

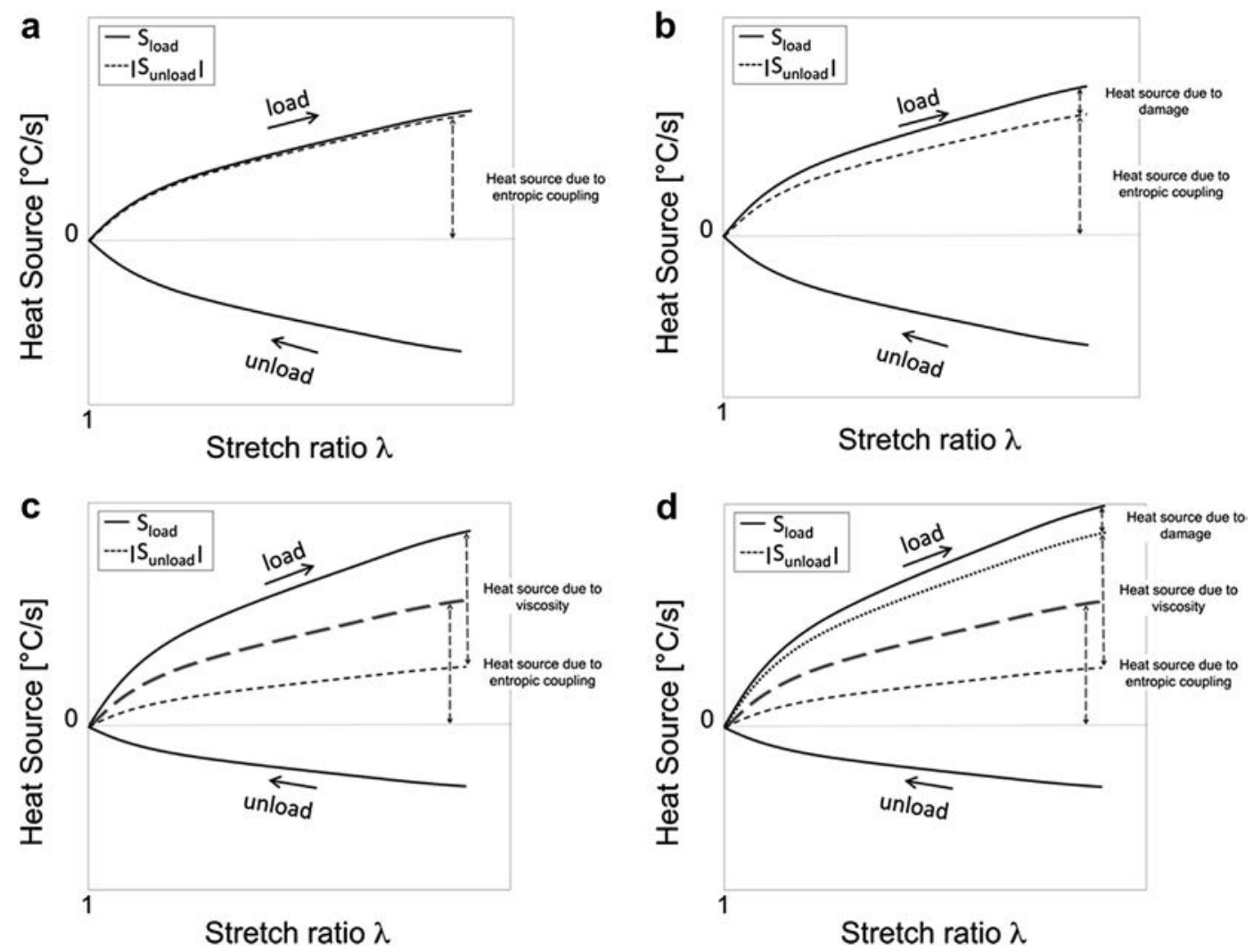

Fig. 7. Schematic evolution: heat source versus stretch ratio for entropic behaviour (a), with damage occurring upon loading (b), with viscosity (c), with damage and viscosity (d).

Table 2

Heat calculated for SBR50.

\begin{tabular}{lrrrc}
\hline & $\begin{array}{c}\text { Cycle 3 } \\
\text { at } \lambda=2\end{array}$ & \multicolumn{1}{c}{$\begin{array}{c}\text { Cycle 3 } \\
\text { at } \lambda=3\end{array}$} & $\begin{array}{c}\text { Cycle 3 } \\
\text { at } \lambda=4\end{array}$ & $\begin{array}{l}\text { Cycle 3 } \\
\text { at } \lambda=4.5\end{array}$ \\
\hline $\mathrm{A}_{\mathrm{n}}=\int \mathrm{S}_{\text {load }} \mathrm{dt}\left({ }^{\circ} \mathrm{C}\right)$ & 0.828 & 2.360 & 4.300 & 5.080 \\
$\mathrm{~B}_{\mathrm{n}}=\int \mathrm{S}_{\text {unload }} \mathrm{dt}\left({ }^{\circ} \mathrm{C}\right)$ & -0.628 & -1.868 & -3.280 & -4.140 \\
$\mathrm{C}_{\mathrm{n}}=\mathrm{A}_{\mathrm{n}}+\mathrm{B}_{\mathrm{n}}\left({ }^{\circ} \mathrm{C}\right)$ & 0.200 & 0.492 & 1.020 & 0.94 \\
$\mathrm{H}_{\mathrm{d} 1}\left(10^{6} \mathrm{~J} / \mathrm{m}^{3}\right)$ & 0.328 & 0.814 & 1.710 & 1.560 \\
\hline
\end{tabular}

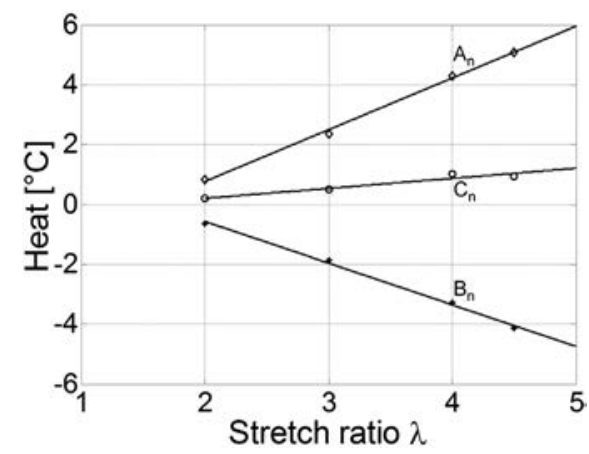

Fig. 8. Heat versus the maximum stretch ratio reached for the third cycle of each of the four series for SBR 50 . cycles. It should be noted that it is not the case for materials whose microstructure significantly evolves during the mechanical cycle, typically crystallisable rubbers [3,4]. Moreover, the higher the stretch ratio, the higher the heat sources produced due to mechanical dissipation. These results constitute an interesting way to validate or enhance constitutive equations.

\section{Conclusions}

Calorimetric effects during SBR deformation processes were investigated using IR thermography measurements, the framework of the thermodynamics of irreversible processes and the heat diffusion equation. More particularly, the effects of the addition of carbon black fillers on the calorimetric response were studied. SBR was tested under cyclic tensile loading conditions. In the present work, only the stabilized response is analysed, i.e. the calorimetric signature of the Mullins effect was not studied here.

Results show that the less-filled SBR exhibits purely entropic behaviour, meaning that no mechanical dissipation is detected. The heat produced and absorbed is the same on loading and unloading at any stretch ratio.

The SBR filled with $50 \mathrm{phr}$ of carbon black behaves as an entropic and viscous material. The mechanical dissipation was calculated. It increases quasi-linearly with the stretch ratio. A simplified framework is proposed to discuss the 
nature and the identification of the mechanical dissipation. This experimental result may be useful for the validation or enhancement of constitutive equations.

\section{Acknowledgements}

The authors would like to acknowledge the "Manufacture Française des Pneumatiques Michelin" for supporting this study. The authors also thank D. Berghezan, F. VionLoisel and E. Munch for their fructuous discussions.

\section{References}

[1] A. Chrysochoos, H. Louche, An infrared image processing to analyse the calorific effects accompanying strain localisation, International Journal of Engineering Science 38 (2000) 1759-1788.

[2] A. Chrysochoos, V. Huon, F. Jourdan, J.-M. Muracciole, R. Peyroux, B. Wattrisse, Use of full-Field digital image correlation and infrared thermography measurements for the thermomechanical analysis of material behaviour, Strain 46 (2010) 117-130.

[3] J.R Samaca Martinez, J.-B. Le Cam, X. Balandraud, E. Toussaint, J. Caillard, Thermal and calorimetric effects accompanying the deformation of natural rubber. Part 1: thermal characterization. http://dx.doi.org/10.1016/j.polymer.2013.03.011.

[4] J.R. Samaca Martinez, J.-B. Le Cam, X. Balandraud, E. Toussaint, J. Caillard, Thermal and calorimetric effects accompanying the deformation of natural rubber. Part 2: quantitative calorimetric analysis, http://dx.doi.org/10.1016/j.polymer.2013.03.012.

[5] Q. Nguyen, P. Germain, P. Suquet, Continuum Thermodynamics (1983).

[6] N. Boccara, Les principes de la thermodynamique classique (1968).

[7] A. Chrysochoos, Analyse du comportement des matériaux par thermographie Infra Rouge, in: Eyrolles (Ed.), Colloque Photomécanique (1995), pp. 203-211.

[8] L. Mullins, Effect of stretching on the properties of rubber, Rubber Chemistry and Technology 21 (1948) 281-300.

[9] J. Diani, B. Fayolle, P. Gilormini, A review on the Mullins effect, European Polymer Journal 45 (2009) 601-612.

[10] D. Favier, L. Orgeas, P. Vacher, L. Meunier, G. Chagnon, Mechanical experimental characterization and numerical modelling of an unfilled silicone rubber, Polymer Testing 27 (2008) 765-777. 